\title{
Biofilm Formation by Enterococcus Faecalis and Enterococcus Faecium: Review
}

\author{
Tsegahun Asfaw* \\ Department of Medical Laboratory Science, Debre Berhan University, Debre Berhan, Ethiopia
}

*Corresponding Author: Tsegahun Asfaw, Department of Medical Laboratory Science, Debre Berhan University, Debre Berhan, Ethiopia

\begin{abstract}
Enterococcus faecalis and Enterococcus faecium are normal intestinal floras of humans and animals. Recently, these organisms' causes serious infections especially in immunocompromised patients and among the leading cause of hospital acquired infections. They are less virulent when compared with other pathogenic organisms. However, E. faecalis and E. faecium biofilms are the major virulence factor of these two species. Biofilms are the population of microorganisms on a biotic or biotic medical devices enclosed by extracellular matrix. They are physiologically different from free flatting bacteria. Biofilms of E. faecalis and E. faecium associated with medical devices are the major contributor in the spread of these organism's infections and antibiotic resistance. In general E. faecalis and E. faecium are strong biofilm producers and biofilms are an important factor in the pathogenesis of these organisms. As a result, patients infected with these organisms' biofilms are very difficult to treat. Hence, the health care workers strongly perform on the infection prevention to prevent medical device mediated E. faecalis and E. faecium biofilm associated infections. Therefore, this review discuses E. faecalis and E. faecium bifolims formation and role in disease pathogenesis.
\end{abstract}

Keywords: Biofilm, Enterococcus faecalis, Enterococcus faecium

\section{INTRODUCTION}

Enterococci are normal (small and large) intestinal flora of human and animal. Recently, these organisms's causes serious infections especially in immunocompromised patients (1). Enterococcus faecalis (E. faecalis) and Enterococcus faecium (E. faecium) are the most dominant species and the leading cause of hospital acquired infections with resistant to multiple antibiotics (2). The most common sites infected by these organisms are urinary tract, blood stream (especially neonate), cardiac, surgical wound, intra-abdominal, pelvic, and rarely the meninges (3).

E. faecalis and E. faecium are less virulent when compared with other pathogenic organisms. The main virulence factors of these organisms are development of multiple antibiotic resistances. These organisms are naturally low level resistant to some antibiotics and high ability to acquire resistant genes (4). In additions, E. faecalis and E. faecium have an ability to form biofilm which increases disease causing ability of these organisms (5).

Biofilms are communities of microorganisms adhering to biological or non biological surfaces enclosed by extracellular matrix and prevalent in hospital settings $(6,7)$. There is an intimate contact within the biofilm microorganisms' cells which increases sharing of their own characteristics. As a result microbial cells growing in a biofilm are physiologically different from free flatting bacteria (8). $E$. faecalis and E. faecium biofilms become one of worldwide problem because of different reasons. Like facilitating spread of antibiotic resistance and mediating medical device associated infections (8). But data about E. faecalis and E. faecium biofilms scare in Africa. So, understanding biofilm formation by enterococci and its role in pathogenesis important for limiting medical device mediated enterococcal infections. Therefore, the objective of this review is to discuss biofilm formation of $E$. faecalis and E. faecium and its role in pathogenesis. 


\section{DISCUSSION}

\subsection{Magnitude of E. Faecalis and E. Faecium Biofim Production}

A study was conducted to show the relationship between biofilm formation, the enterococcal surface protein (Esp) and gelatinase. Among 128 clinical isolates of enterococci 95.2\% E. faecalis and 28.8\% E. faecium were form biofilm (9). A study was conducted between enterococcal biofilm formation in vitro and medical device related infection potential in vivo showed about 109 enterococci were isolated from blood and $100 \%$ of E. faecalis and $42 \%$ E. faecium isolates were formed biofilms (10). Another study had shown E. faecalis biofilms from endocarditis and non-endocarditis isolates, $39 \%$ and $6 \%$ were produced strong biofilm, respectively (11).

A research was conducted to see the influence of origin of isolate in E. faecalis biofilm formation. Among 79 E. faecalis isolates from endocarditis, 31(37.2\%), 41(52\%), 7(9\%) were strong, medium and weak biofilm producers, respectively. On the other hand, among 22 E. faecalis isolates from urine, $0(0 \%), 17(21.5 \%), 3(6.3 \%)$, and 2(2.5\%) were strong, medium, weak and non-biofilm formers, respectively. Moreover, Among 31 E. faecalis isolates from other clinical specimen except blood and urine, 2(6.5\%), 17(54.8\%), 6(19.4\%), 6(19.4\%) were strong, medium, weak and non-biofilm formers, respectively (11).

A study was conducted to see biofilm formation by multidrug resistant E. faecalis from clinical isolates. Among 40 multi-drug resistant E. faecalis strains, 27.5\%, 55\%, 17.5\% were strong, moderate and weak or non-biofilm producers, respectively (12). Another study was conducted among clinical and commensal isolates of E. faecalis for presence of Enterococci Surface Protein (esp) gene and biofilm production. Among 200 clinical isolates $32.5 \%$ were positive for biofilm production and from these $30 \%$ were positive for the esp gene. On the other hand among 100 commensal isolates $8 \%$ and $147 \%$ were positive for biofilm formation and the esp gene, respectively (13).

\subsection{Clinical Significances}

E. faecalis and E. faecium biofilms pose a major challenge and account for most infections especially, in the case of patients under treatment with indwelling medical devices (8). High prevalence of $E$. faecalis and E. faecium urinary catheter associated infections, central venous catheter associated bacteremia and endocarditis involves the formation of biofilm (14). It has been estimated that over $80 \%$ of enterococci nosocomial infections are derived from biofilm related infections (15).

\subsection{Steps of Biofilm Formation}

The basic structural unit of the biofilm is the microcolony. Proximity of cells within the microcolony (or between microcolonies) provides an ideal environment for creation of nutrient gradients, exchange of genes, and quorum sensing (16). The formation of biofilm is start with adherence of the free floating cell to biotic or non biotic surface. Adherence cells are followed by a reversible attachment (17). Then the irreversible attachment occurs as a result of concurrent production of the extracellular polymeric substances (EPS). The maturation of biofilm attained when the irreversible attached cells developed to form a more organized and complex structure and shape depends on source of the nutrients. An established biofilm structure comprises microbial cells and EPS (8).

Dispersion is the movement of organisms away from their place of population density (biofilm). Biofilm cells may be dispersed in different ways: 1) Shedding of daughter cells from actively growing cells, 2) Detachment as a result of nutrient levels or quorum sensing, 3) Shearing of biofilm aggregates (continuous removal of small portions of the biofilm) because of flow effects. The rate of dispersion increases with increase in biofilm thickness and nutrient or oxygen depletion $(7,18)$. Figure 2 show pictorially stages of biofilm formation.

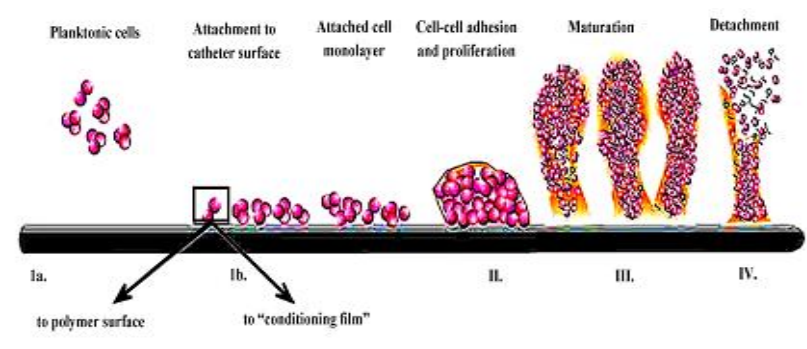

Figure2. Stages of biofilm formations (8). 


\subsection{Biofilm in Disease Pathogenesis}

Infections associated with biofilm initially confined to a particular location and though time detachment may occur. However, the detached biofilms may result in bloodstream or urinary tract infections or in the production of blockage of blood flow (emboli) (19). On the other hand, cells in biofilms are mostly resistant to antimicrobial agents and the host immune system. Biofilms formed by E. faecalis are 1000 times more resistant to phagocytosis, antibodies and antimicrobial agents than non-biofilm producers. Therefore, infections of E. faecalis and E. faecium associated with biofim aggravated in this case $(17,20)$.

In the case of endocarditis a complex biofilm composed of either E. faecalis or E. faecium and host components will be formed on a cardiac valve. These biofilms causes disease by three basic mechanisms. First, the biofilms physically disrupts valve function and may cause leakage. Second, detachment of biofilm can be carried to a terminal point in the circulation and formation of emboli (blockage of the blood vessel. Third, the biofilm provides continuous infection of the bloodstream even during antibiotic treatment. These can causes recurrent fever, chronic systemic inflammation, and other infections $(11,17)$. Figure 1crearly shows how biofilm mediate an infection.

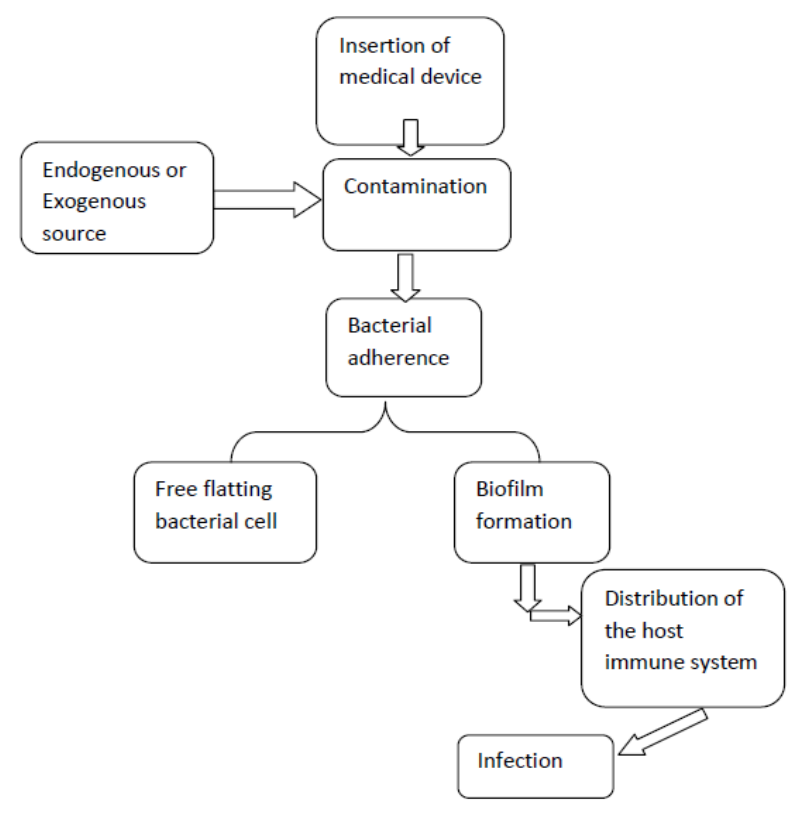

Figure1. Schematic flow of biofilm mediated infections (8).

\subsection{Factors Influencing Biofilm Formation}

\subsubsection{Biological}

Biofilm formation is a complex process regulated by diverse characteristics of the cell. Property of cell such as cell surface, hydrophobicity, adhesion proteins, surface charge, and extracellular polymeric substances influence attachment and coaggregation of E. faecalis and E. faecium biofilm formation (20). Studies had shown that the contribution of esp on biofilm formation of enterococci contradicted. A study was conducted to see the relationship between Esp and E. faecalis biofilm formation. However, the result of the study had shown ESP promotes primary attachment and biofilm formation of E. faecalis on the surfaces $(15,20)$. Another study had shown that the independency of ESP for E. faecalis biofilm formation (21). Similar study was conducted to show the relationship between ESP and E. faecium E1162. Enterococcal surface protein (ESP) had an important role in biofilm formation for E. faecium E1162 strain (22). In addition, table1 shows different genes involved in E. faecalis and E. faecium biofilm formation.

Table1. Genes involved in E. faecalis and E. faecium biofilm formation

\begin{tabular}{|c|c|c|c|c|}
\hline Type of gene & Name of protein & Function & Species & Reference \\
\hline $\begin{array}{c}\text { fsrA, fsrB, and } \\
\text { fsrC }\end{array}$ & $\begin{array}{c}\text { serine protease(spr)E } \\
\text { and gelatinase(GelE) }\end{array}$ & $\begin{array}{c}\text { quorum sensing \& autolysis } \\
\text { (release f eDNA) })\end{array}$ & $\begin{array}{c}\text { E. faecalis and } \\
\text { E. faecium }\end{array}$ & $(23,15,22)$ \\
\hline esp & $\begin{array}{c}\text { Enterococcal surface } \\
\text { protein }\end{array}$ & Attachment & $\begin{array}{c}\text { E. faecalis and } \\
\text { E. faecium }\end{array}$ & $(20,22,24)$ \\
\hline
\end{tabular}




\begin{tabular}{|c|c|c|c|c|}
\hline epa (orfde5) & $\begin{array}{c}\text { enterococcal } \\
\text { polysaccharide antigen }\end{array}$ & $\begin{array}{c}\text { attachment or biofilm } \\
\text { accumulation }\end{array}$ & E. faecium & $(11)$ \\
\hline Asc10 & ASC10 & Aggregation & E. faecalis & $(25)$ \\
\hline$C y l A$ & $C y l$ & Cytolysin & E. faecalis & $(26)$ \\
\hline Acc(6')/aph(2") & Acc(6')/aph(2") & In activating antibiotics & E. faecalis & $(26)$ \\
\hline
\end{tabular}

\subsubsection{Environmental}

The physicochemical properties of the surface may exert a strong influence on the rate and extent of attachment. Temperature, cations, and presence of antimicrobial agents influence the attachment. Glucose is the major determinate in the formation of E. faecalis and E. faecium biofilm (27). Host produced conditioning films such as blood, tears, urine, saliva, intravascular fluid, and respiratory secretions influence the attachment of E. faecalis and E. faecium (19).

A research indicates that the presence of Streptococcus mutans increases E. faecalis biofilm formation (28). Biofilm productions of both E. faecalis and E. faecium at $37^{\circ} \mathrm{c}$ obviously increases (5). E. faecalis and E. faecium can produce biofilm at $4.5 \mathrm{pH}$ but more biofilm production is at $8.5 \mathrm{pH}$. On the contrary, increment in salt concentration decrease biofilm production (29).

\subsection{Detection of Biofilm}

Different phenotypic techniques including: test tube method, tissue culture plate method, Congo red agar and Microtiter dish biofilm formation assay used to detect enterococcal biofilm $(12,30)$. Tissue culture plate method is more accurate and sensitive to detect $E$. faecalis biofilm (16). Microtiter dish biofilm formation assay is an important to test early stage of biofilm. This assay works by allowing the formation of biofilm on the wall or bottom of Micro titer dish (30). Except Congo red agar all the above assays need staining. In order to see the presence of biofilm different days can be used example: acridine orange and crystal violet. Congo red agar method detect by culturing from another culture and produces different colure used for identification. The biofilm formers produce black colonies and the non formers remain pink (30).

\subsection{Prevention and Control}

Infections caused by E. faecalis and E. faecium biofilms are very difficult to treat. However, prevention of biofilms formation by these organisms is very important (31). One of the best ways to eliminate biofilm is preventing the initial attachment of E. faecalis and E. faecium cells by avoiding contamination of the exposed surface. In addition, prevention at any stages of biofilm development is also possible and important (32).

Control of biofilm to prevent further contamination is important to prevent further infection. $2.5 \%$ and $5.25 \%$ sodium hypochlorite were the most effective irrigates to eliminate E. faecalis biofilms (32). Chlorhexidine is also an effective irrigates to eliminate almost all strains of E. faecalis (33). A research had been shown that in vitro honey is effective in reducing established biofilm for most all strains of enterococci (34).

\section{CONCLUSION AND RECOMMENDATIONS}

The highest degree of biofilm formation commonly found in isolates of endocarditis followed by urinary tract clinical isolates. Different biological and environmental factors are involved in the formation of E. faecalis and E. faecium biofilm formation. Different researches have contradicted information about esp is a determinate factor or not on the biofilm formation of E. faecalis and $E$. faecium. More E. faecalis and E. faecium biofilm production have been seen at alkaline $\mathrm{pH}$ and at low salt concentration. In addition, glucose is a major contributor in the production of $E$. faecalis and $E$. faecium biofilms. $2.5 \%$ and $5.25 \%$ sodium hypochlorite, Chlorhexidine and honey are effective irrigates to eliminate established enterococci biofilms. In general biofilms of E. faecalis and $E$. faecium are an important factor in the pathogenesis. Literatures showed that infections of E. faecalis and E. faecium are mainly due to medical device mediated associated with biofilm formation. Decreasing ability to prevent biofilm formations makes: the treatment and control of E. faecalis and $E$. faecium infections very difficult. Therefore, the health care workers strongly perform on the infection prevention to prevent medical device mediated E. faecalis and E. faecium biofilm associated infections and further researches need to conduct on the area of the exact factors and mechanisms involved in biofilm production by E. faecalis and E. faecium. Moreover, its role in disease pathogenesis. 


\section{REFERENCES}

[1] Fisher K, Phillips C. Ecology, epidemiology and virulence of enterococcus. Microbiol.2009; 155(6):17491757.

[2] Akhi M, Farzaneh F, Oskouei M. Study of enterococcal susceptibility patterns isolated from clinical specimens in Tabriz, Iran. Pak J Med Sci. 2009; 25(2): 211-216.

[3] Sood S. Malhotra M, Das B, Kapil A. Enterococcal infections and antimicrobial resistance. Indian J Med Res.2008; 128(2): 111-121.

[4] Fernandes S, Dhanashree B. Drug resistance and virulence determinants in clinical isolates of Enterococcus species. Indian J Med Res.2013; 137(5): 981-985.

[5] Marinho A, Martins P, Ditmer E, Azevedo P, Frazzon J, Sand S, Frazzon A. Biofilm formation on polystyrene under different temperatures by antibiotic resistant E. faecalis and Enterococcus faecium isolated from food. Brazil J Microbiol.2013; 44(2)423-426.

[6] Bose S, Krishna A. Biofilms: A challenge to medical science. J Clin Diag Res. 2011; 5(1):127-130.

[7] Mohammed J, Mohammed B, Hindatu Y, Sulaiman M, Saidu H, Idris Abdulrahman, Tijani H. Bacterial biofilm: A Major Challenge of Catheterization. J Microbiol Res.2013; 3(6): 213-223.

[8] Stewart P, Franklin M. Physiological heterogeneity in biofilms. Nature Rev Microbiol.2008; 6(3):199-210.

[9] Rosa , R, Creti R, Venditti M, Amelio R, Arciola C, Montanaro L etal. Relationship between biofilm formation, the enterococcal surface protein (Esp) and gelatinase in clinical isolates of E. faecalis and $E$. faecium. FEMS Microbiol Lett.2006; 256: 145-150.

[10] Jonathan A, Sandoe T, Ian R, Jonathan W, Itherden H, Heritage C, Wilcox M. Correlation between enterococcal biofilm formation in vitro and medical-device-related infection potential in vivo. $\mathrm{J}$ Med Microbiol.2003; 52: 547-550.

[11] Mohamed J, Huang W, Nallapareddy S, Teng F, Murray B. Influence of origin of isolates, especially Endocarditis Isolates, and various genes on biofilm formation by E. faecalis. Infect Immun.2004; 72(6):3658-3663.

[12] Oli A, Rajeshwari R, Chandrakanth N. Biofilm formation by Multidrug resistant E. faecalis (MDEF) originated from clinical samples. J Microbiol Biotech Res.2012; 2(2):284-288.

[13] Upadhyaya G, Lingadevaru U, Lingegowda R. Comparative study among clinical and commensal isolates of E. faecalis for presence of esp gene and biofilm production. J Infect Dev Ctries.2011; 5(5):365-369.

[14] Pillai S, Sakoulas G, Eliopoulos G, Moellering R, Murray B, Inouye R. Effects of Glucose on $f s r$-Mediated Biofilm Formation in E. faecalis. J Infect Dis. 2004; 190:967-970.

[15] Arana A, Valle J, Solano C, Arrizubieta M, Cucarella C, Lamata M etal. The Enterococcal Surface Protein, Esp, Is Involved in E. faecalis Biofilm Formation. App Environ Microbiol.2001; 67(10):4538-4545.

[16] Kour M, Somi I, Sharma R. Assessment of biofilm formation by E. faecalis causing nosocomial infections and their statistical analysis. Indian J Appl Res.2014; 4(1):77-79.

[17] Oliveira M, Santos V, Fernandes A, Bernardo F, Vilela C. Antimicrobial resistance and in vitro biofilmforming ability of enterococci from intensive and extensive farming broilers. Poultry Science.2010; 89:1065-1069.

[18] Usha H, Kaiwar A, Mehta D. Biofilm in endocarditis: New understanding to an old problem. Int $J$ Contemporary Dentistry.2010; 1(3): 44-51.

[19] Parsek M, Singh P. Bacterial biofilms: An emerging link to disease pathogenesis. Annu Rev Microbiol.2003; 57: 677-701.

[20] Donlan R. Biofilms: Microbial life on surfaces. Emerging Infect Dis.2002; (8)9: 881- 890.

[21] Tendolkar P, Baghdayan A, Gilmore M, Shankar N. Enterococcal surface protein, Esp, enhances biofilm formation by E. faecalis. Infect Immun.2004; 72(10):6032-6039.

[22] 22 Thomas V, Thurlow L, Boyle D, Hancock L. Regulation of Autolysis-Dependent Extracellular DNA Release by E. faecalis Extracellular Proteases Influences Biofilm Development. J Acteriol.2008; 190(16): 5690-5698.

[23] Kristich C, Li Y, Cvitkovitch D, Dunny G. Esp-Independent biofilm formation by E. faecalis. J. Bacteriol.2004; 186(1):154-163.

[24] Heikens E, Marc M, Willems B. Enterococcal surface protein Esp is important for biofilm formation of $E$. faecium E1162. J Bacteriol.2007; 189(22):8233-8240.

[25] Chuang-Smith O, Wells C, Henry-Stanley M, Dunny G. Acceleration of E. faecalis biofilm formation by aggregation substance expression in an ex vivo model of cardiac valve colonization. PLoS.2010; (5)12: 15798. 
[26] Moniri R, Ghasemi A, Moosavi S, Dastehgoli K, Rezaei M. Virulence gene's relationship with biofilm formation and detection of aac (6')/aph (2") in E. faecalis isolated from patients with Urinary Tract Infection. Jundishapur J Microbiol. 2013; 6(5): 6244.

[27] Mohamed J, Huang B. Biofilm formation by enterococci. J Med Microbiol.2007; 56: 1581-1588.

[28] Deng D, Hoogenkamp A, Exterkate R, Jiang L, van der Sluis L, ten Cate J, Crielaard W. Influence of Streptococcus mutans on E. faecalis biofilm formation. J Endod.2009; 35(9):1249-1252.

[29] Peter A, Zacharia S, Mathew J. Biofilm formation in enterococci from different sources. Int $J$ Biopharmaceutics. 2013; 4(2): 140-144.

[30] Niveditha S, Pramodhini S, Umadevi S, Kumar S, Stephen S. The isolation and the biofilm formation of uropathogens in the patients with catheter associated Urinary Tract Infections (UTIs). J Clin Diag Res. 2012; 6(9): 1478-1482.

[31] Dová N, Janštová S, Karpíšková S, Dušková C, Karpíšková M. Importance of Enterococcus spp for forming a biofilm. Czech J Food Sci.2009; 27:354-356.

[32] Murad C, Sassone L, Souza M, Sergio R, Fidel S. Antimicrobial activity of sodium hypochlorite, chlorhexidine and MTAD against E. faecalis biofilm on human dentin matrix in vitro. RSBO.2012; 9(2):143-150.

[33] Arias-Moliz M, Ferrer-Luque C, González-Rodríguez M, Navarro-Escobar E, Freitas M. Antimicrobial activity and E. faecalis biofilm formation on chlorhexidine varnishes. Med Oral Patol Oral Cir Bucal.2012; 17 (4):705-709.

[34] Lim KY, Chong JY, Low KL. In vitro Screening of Honey against Enterococcus spp. Biofilm. J Med Bioengineering.2014; 3(1): 23-28.

Citation: Tsegahun Asfaw, "Biofilm Formation by Enterococcus Faecalis and Enterococcus Faecium: Review" International Journal of Research Studies In Biosciences (Ijrsb), Vol. 7, no. 4, pp. 5-10, 2019. http://Dx.Doi.org/10.20431/2349-0365.0704002

Copyright: (C) 2019 Authors. This is an open-access article distributed under the terms of the Creative Commons Attribution License, which permits unrestricted use, distribution, and reproduction in any medium, provided the original author and source are credited. 